\title{
An N-terminal-truncated isoform of FAM134B (FAM134B-2) regulates starvation-induced hepatic selective ER-phagy
}

\author{
Shohei Kohno, Yuji Shiozaki, Audrey L Keenan, Shinobu Miyazaki-Anzai, Makoto Miyazaki (i)
}

\begin{abstract}
Autophagy is a conserved system that adapts to nutrient starvation, after which proteins and organelles are degraded to recycle amino acids in response to starvation. Recently, the ER was added to the list of targets of autophagic degradation. Autophagic degradation pathways of bulk ER and the specific proteins sorted through the ER are considered key mechanisms in maintaining ER homeostasis. Four ER-resident proteins (FAM134B, CCPG1, SEC62, and RTN3) have been identified as ER-resident cargo receptors, which contain LC3-interacting regions. In this study, we identified an N-terminal-truncated isoform of FAM134B (FAM134B-2) that contributes to starvation-induced ER-related autophagy. Hepatic FAM134B-2 but not full-length FAM134B (FAM134B-1) is expressed in a fed state. Starvation drastically induces FAM134B-2 but no other ER-resident cargo receptors through transcriptional activation by C/EBP $\beta$. C/EBP $\beta$ overexpression increases FAM134B-2 recruitment into autophagosomes and lysosomal degradation. FAM134B-2 regulates lysosomal degradation of ER-retained secretory proteins such as ApoCIII. This study demonstrates that the C/EBP $\beta$ FAM134B-2 axis regulates starvation-induced selective ER-phagy.
\end{abstract}

DOI 10.26508/lsa.201900340 | Received 11 February 2019 | Revised 7 May 2019 | Accepted 7 May 2019 | Published online 17 May 2019

\section{Introduction}

The ER is the most abundant membrane structure in the cell. The ER is the central organelle that regulates protein synthesis and modification, lipid metabolism, and calcium homeostasis (Borgese et al, 2006; Braakman \& Hebert, 2013; Krebs et al, 2015). Differences in physical and functional characteristics distinguish the two types of ER, known as rough ER (RER) and smooth ER (SER). RER attaches to ribosomes to synthesize proteins, whereas SER synthesizes lipids (Borgese et al, 2006). Two major pathways in the $E R$, the unfolded protein response and ER-associated protein degradation (ERAD), are known to control ER homeostasis when ER perturbations occur. Unfolded protein response activation increases the folding capacity of the ER, whereas the ERAD system recognizes terminally misfolded proteins and degrades them by the ubiquitin-proteasome system (Hwang \& Qi, 2018; Kroeger et al, 2018). These two pathways maintain the flow of synthesis, folding and clearance of ER-resident proteins. In addition to the two ER homeostasis pathways, recent studies revealed the ER as a new target of autophagic degradation (Mochida et al, 2015). ER-resident membrane proteins family with sequence similarity 134 member $B$ (FAM134B), preprotein translocation factor (SEC62), cell cycle progression protein 1 (CCPG1), and reticulon 3 (RTN3) were identified as ER-phagy receptors (Khaminets et al, 2015; Fumagalli et al, 2016; Grumati et al, 2017; Fregno \& Molinari, 2018; Smith et al, 2018). These proteins contain LC3-interacting regions in a cytosolic domain and mediate autophagic cargo followed by lysosomal degradation.

The liver is a central organ that regulates nutrient and drug metabolisms in response to various nutritional stresses. Nutrient starvation is a major trigger regulating several adaptive changes in the liver, including macroautophagy (Komatsu et al, 2005; Ezaki et al, 2014; Fullgrabe et al, 2016). Starvation-induced hepatic autophagy is regulated by transcription factors and nutrient-sensing kinases at the transcriptional and post-translational levels, respectively (Goldstein \& Hager, 2015; Ueno \& Komatsu, 2017). At the transcriptional level, a number of transcription factors contribute to starvation-induced autophagy gene expression, such as CREB, PPARs, FXR, FOXOs, and C/EBPS (Goldstein \& Hager, 2015). Starvation is also known to induce autophagy-mediated ER degradation (Komatsu et al, 2005). However, which ER-resident cargo receptor contributes to starvation-induced hepatic ER-phagy and which transcription factor regulates starvation-induced ER-related selective autophagy have not been elucidated.

In this study, we identified a novel N-terminal-truncated isoform of FAM134B (FAM134B-2) as a starvation-induced ER-resident cargo receptor. Starvation selectively induced FAM134B-2 gene expression through the induction of $C / E B P \beta$ and also increased the recruitment of FAM134B-2 into autophagosomes and lysosomal degradation of FAM134B-2 in vivo and in vitro. FAM134B-2 was localized in both the SER and RER. In addition, FAM134B-2 regulates the selective ER-phagy of secretary proteins such as apolipoprotein C-III (ApoCIII) but not bulk ER turnover. These data suggest that FAM134B-2 is a major contributor to starvation-induced selective ER-phagy.

Division of Renal Diseases and Hypertension, Department of Medicine, University of Colorado Denver, Aurora, CO, USA 


\section{Results}

\section{Selective induction of a truncated isoform of FAM134B (FAM134B-2) by starvation}

We performed quantitative reverse transcription PCR (qRT-PCR) to determine which hepatic ER-resident cargo receptor is transcriptionally regulated upon starvation. $16 \mathrm{~h}$ of fasting selectively increased mRNA levels of FAM134B, which were normalized by $6 \mathrm{~h}$ of re-feeding (Fig 1A). Hepatic mRNA expression of the other ER-phagy receptors (SEC62, CCPG1, and RTN3) was not altered by fasting and re-feeding (Fig 1A). We next determined whether starvation increases levels of FAM134B protein. To identify FAM134B proteinspecific signal in the liver, we used two references: 1 ) protein lysates from the mouse brain, which highly expresses FAM134B and 2) recombinant mouse FAM134B protein generated in HEK293T cells. Immunoblot analysis showed that the mouse liver did not express full-length FAM134B (FAM134B-1) in either the fed or fasted states, as described recently by Schultz et al (2018). Interestingly, however, the smaller size of FAM134B (FAM134B-2) was only detected in the livers of fasted mice (Fig 1B). Although the expected sizes of FAM134B-1 and FAM134B-2 are $\sim 50$ and $\sim 39 \mathrm{KD}$, respectively, on the SDS-PAGE, FAM134B-1, and FAM134B-2 migrated to $\sim 65$ and $\sim 50 \mathrm{KD}$, respectively. This is probably due to post-translational mechanisms. The antibody recognizes the $\mathrm{C}$-terminal region of the FAM134B protein, suggesting that the truncation occurs at the $\mathrm{N}$-terminal of the protein. Because FAM134B-1 was not detected in the liver, the truncation was probably not due to the posttranslational proteolytic cleavages. We, therefore, hypothesized that another isoform of FAM134B mRNA occurs through alternative transcription. To identify the alternative transcript of FAM134B, we performed 5'-rapid amplification of CDNA ends (RACE) using CDNA from fasted mouse liver; brain cDNA was used as a reference. The 5'RACE analysis showed that liver and brain cDNA produced different sizes of the N-terminal FAM134B DNA fragments (Fig 1C). The CDNA sequences of FAM134B amplified by 5'RACE are shown in

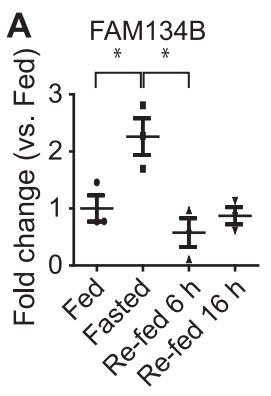

B

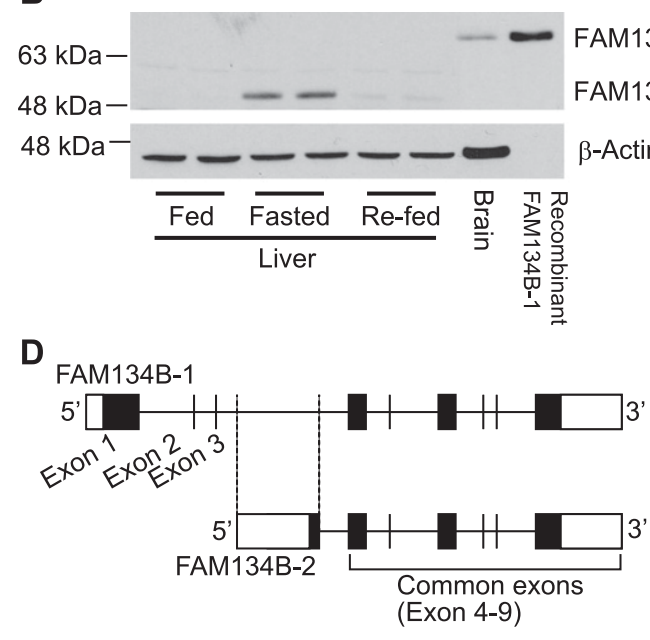

$\mathbf{F}$

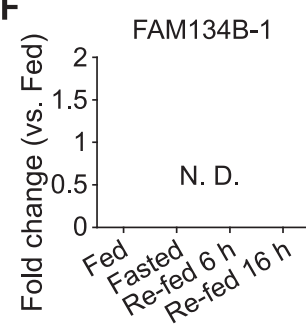

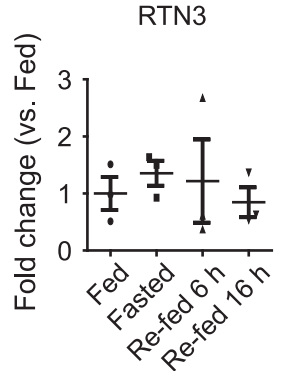

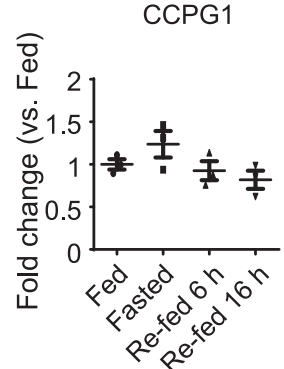

C

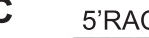

5'RAC

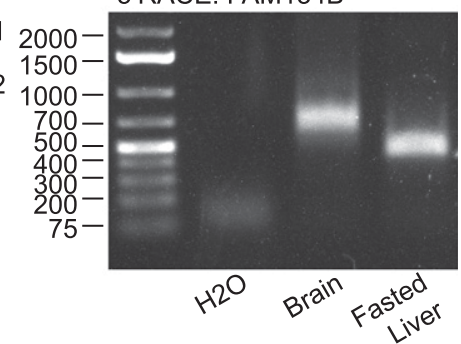

E
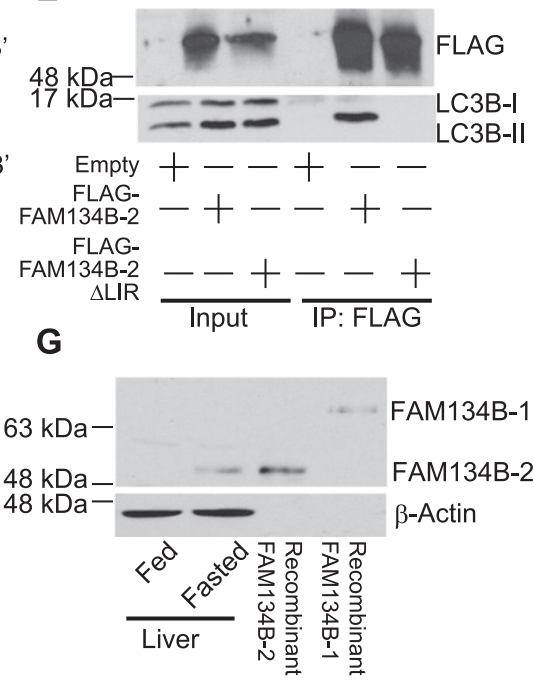

Figure 1. Starvation triggers the selective induction of FAM134B-2.

(A) qRT-PCR analysis of FAM134B, SEC62, RTN3, and CCPG1 in mouse livers from fed, fasted, and re-fed conditions. (B) Immunoblot analysis of FAM134B in mouse livers (fed, fasted, and re-fed), mouse brain, and recombinant FLAG-FAM134B-1 generated in HEK293T cells. (C) Gel electrophoresis of 5' RACE products using brain CDNA from fed mice or liver CDNA from fasted mice. (D) Schematic models of mouse FAM134B-1 and FAM134B-2 genes. (E) Co-immunoprecipitation of endogenous LC3B, FLAG-FAM134B-2, and FLAG-FAM134B2- $\triangle$ LIR. FLAG-FAM134B-2 was transiently overexpressed in HEK293T cells. $24 \mathrm{~h}$ after transfection, the cells were starved for $6 \mathrm{~h}$ in the presence of EBSS and bafilomycin A1 $(1 \mu \mathrm{M})$. (F) qRT-PCR analysis of FAM134B-1 and FAM134B-2 in mouse livers under fed, fasted, and re-fed conditions. (G) Immunoblot analysis of FAM134B in mouse livers (fed and fasted), recombinant FLAGFAM134B-1 and 2 generated in HEK293T cells. One-way ANOVA with a Student-Newman post hoc test was used for statistical analysis. ${ }^{\star} P<0.05$. 
Fig S1. Fig 1D shows the alternative forms of exon 1 in FAM134B. The novel truncated isoform of the FAM134B gene (FAM134B-2) consists of 6 exons which encode 356 amino acids, whereas FAM134B-1 consists of 9 exons which encode 480 amino acids (Fig 1D). The exon 1 of FAM134B-2 splices into the exon 4 of FAM134B-1 (Fig 1D). An analysis using the network-based algorithm Protter (http:// wlab.ethz.ch/protter/start/) predicted that FAM134B-2 is a type I membrane protein consisting of one transmembrane domain (amino acids 55-88), whereas FAM134B-1 has four transmembrane domains. More importantly, FAM134B-2 preserves the cytosolic LC3interacting region but partially lacks the reticulon homology domain, both of which are essential for this function (Fig S2B) (Khaminets et al, 2015). The structures of the human FAM134B-2 gene and protein are very similar to those of mouse FAM134B-2 (Fig S2A and B). We, therefore, performed co-immunoprecipitation using FLAG-tagged FAM134B-2 expressing HEK293T cells to determine the interaction between FAM134B-2 and LC3B. The immunoprecipitant with FLAG antibody contains LC3B-II, whereas the deletion of the LIR of FAM134B-2 abolishes the interaction with LC3B-II (Fig 1E). The data suggest that FAM134B-2 interacts with LC3B-II and the reticulon homology domain may not be essential for this function. To separately quantify levels of FAM134B isoforms, we designed specific primers for each isoform as shown in Fig S2C and Table S1. The qRT-PCR primer pairs specifically amplified the target isoforms (Fig S2C). FAM134B-1 mRNA expression was detected in the brain but not in the fasted liver, whereas FAM134B-2 mRNA expression was detected in the liver but not in the brain (Fig S2C). Hepatic FAM134B-1 mRNA was undetectable in both fed and fasted states (Fig 1F). Starvation selectively induced mRNA levels of hepatic FAM134B-2, consistent with the immunoblot analysis (Fig 1F). Re-feeding normalized starvation-induced FAM134-2 expression (Fig 1F). The immunoblot analysis confirmed that the size of the smaller FAM134B signal induced by starvation exactly matched that of recombinant FAM134B-2 protein but not FAM134B-1 (Fig 1G). We next analyzed whether starvation induces FAM134B-2 in other organs. As shown in Fig S2D, starvation induced FAM134B-2 expression in peripheral organs such as the kidney, spleen, and white adipose tissue but not the brain, where FAM134B-1 is highly expressed.

\section{C/EBP $\beta$ mediates starvation-induced FAM134B-2 expression}

Because levels of FAM134B-2 mRNA were increased under starvation, we thought that starvation regulates FAM134B-2 expression during transcription. To elucidate which starvation-regulating transcription factor induces FAM134B-2 transcription, 1.5-kb FAM134B-2 promoter was cloned to a luciferase reporter vector, pGL3, which was transfected onto HEK293T cells with 19 known transcription factors involved in fasting and starvation (Goldstein \& Hager, 2015). As shown in Fig $2 \mathrm{~A}, \mathrm{C} / \mathrm{EBP} \alpha$ and $\mathrm{C} / \mathrm{EBP} \beta$ but not the other transcription factors significantly increased the promoter-driven luciferase activity by 16 and 9-fold, respectively, compared with empty vector. We next confirmed whether starvation induces levels of hepatic C/EBP $\alpha$ and C/EBP $\beta$. qRT-PCR and immunoblot analysis showed that C/EBP $\beta$ (both liver-enriched transcriptional activator protein [LAP] and liverenriched transcriptional inhibitory protein [LIP]) but not C/EBP $\alpha$ expression was induced in the livers of fasted mice (Fig 2B-F). Starvation-induced C/EBP $\beta$ expression was normalized by re-feeding
(Fig 2C, D, and F). Starvation-induced levels of C/EBP $\beta$ in the kidney, spleen, and white adipose tissue, whereas the brain expressed $\mathrm{C}$ / EBP $\beta$ at very low levels (Fig S3A). To find which region of the FAM134B-2 promoter is responsible for C/EBP $\beta$-mediated FAM134B-2 induction, a promoter deletion assay was performed. C/EBP $\beta$ overexpression increased 1.5, 0.5, and 0.2 kb FAM134B-2 promoter-derived luciferase activity by sixfold, sixfold, and fourfold, respectively, whereas the 0.1-kb promoter was not activated (Fig $2 \mathrm{G}$ ). These results suggest that the $C / E B P \beta$-responsive element should be located between positions -500 to -100 of the FAM134B-2 promoter. A Webbased computational analysis (www.gene-regulation.com) of the FAM134B-2 promoter revealed three putative C/EBP $\beta$-binding sites (Fig $2 \mathrm{H}$ ). To demonstrate which site mediates the effect of $\mathrm{C} / \mathrm{EBP} \beta$ on the FAM134B-2 promoter, mutations in all three putative sites were generated in the pGL3 containing 1.5-kb FAM134B-2 promoter and subjected to the same transfection assay. Mutations in the -154 to -141 positions of the FAM134B-2 promoter but no other mutations significantly reduced the response to C/EBP $\beta$ overexpression (Fig $2 \mathrm{H}$ ). Together, these results indicate that a $C / E B P \beta$ responsive element is located in the FAM134B-2 promoter between positions -154 to -141 . To confirm that $C / E B P \beta$ physiologically binds to the $C / E B P \beta$ responsive element of the FAM134B-2 promoter, we performed chromatinimmunoprecipitation (ChIP) assays in the livers from fed and fasted animals. The ChIP-RT-qPCR assay confirmed that starvation significantly increased the recruitment of $\mathrm{C} / \mathrm{EBP} \beta$ but not C/EBP $\alpha$ onto the FAM134B-2 promoter in vivo compared with the fed state (Fig $2 \mathrm{I}$ and J). To examine whether $C / E B P \beta$ is fully responsible for the induction of hepatic FAM134B-2 expression, we generated liver-specific human Myc$C / E B P \beta$ (also called LAP) transgenic mice (Fig 3A). To eliminate undesirable effects by random insertion of a transgene into the genome, floxed human Myc-C/EBP $\beta$ was inserted into a known permissive locus, Rosa26, by a recombinase-mediated cassette exchange procedure that we previously developed and have used for other transgenic lines. Rosa- $h C / E B P \beta$ mice were crossed with Albumin (Alb)-Cre mice. Alb-Cre; Rosa-hC/EBP $\beta$ mice expressed Myc-hC/EBP $\beta$ only in the liver (Fig S3B), which increased the levels of hepatic total $C / E B P \beta$ protein under the fed state compared with Alb-Cre (control) littermates (Fig 3C). The overexpression of hepatic C/EBP $\beta$ induced mRNA and protein expression of FAM134B-2 (Fig 3B and C) but no other ER-phagy receptors (Fig S3C-F). C/EBP $\beta$ overexpression also increased levels of hepatic ATG5 and LC3B-II protein, suggesting that overall autophagy is induced by $\mathrm{C} / \mathrm{EBP} \beta$ overexpression (Figs $3 \mathrm{C}$ and $\mathrm{S} 3 \mathrm{G}$, and $\mathrm{H}$ ). Injections of leupeptin (an inhibitor of lysosomal cysteine, serine, and threonine peptidases) increased FAM134B-2 protein levels in L-C/EBP $\beta$ mice (Fig $3 D$ ). To further confirm the $C / E B P \beta$-mediated induction of FAM134B-2, we generated liver-specific C/EBP $\beta$ KO (Alb-Cre $(+) ; C / E B P \beta^{\text {flox/flox } L-C / ~}$ EBP $\beta$ KO) mice by crossing Alb-Cre and $C / E B P \beta$-floxed mice. We used Alb-Cre(-); C/EBP $\beta^{\text {flox/flox }}$ mice as a control. Liver-specific C/EBP $\beta$ deficiency significantly blocked starvation-induced FAM134B-2 expression (Fig 3E and F).

\section{FAM134B-2 is expressed in the SER and RER}

We next investigated whether FAM134B-2 protein is localized in a specific ER compartment using iodixanol density gradient ultracentrifugation (Opti-Prep) with hepatic microsomes of fasted mice. Sarco/endoplasmic reticulum $\mathrm{Ca}^{2+}$-ATPase-2 (SERCA2) and 
A

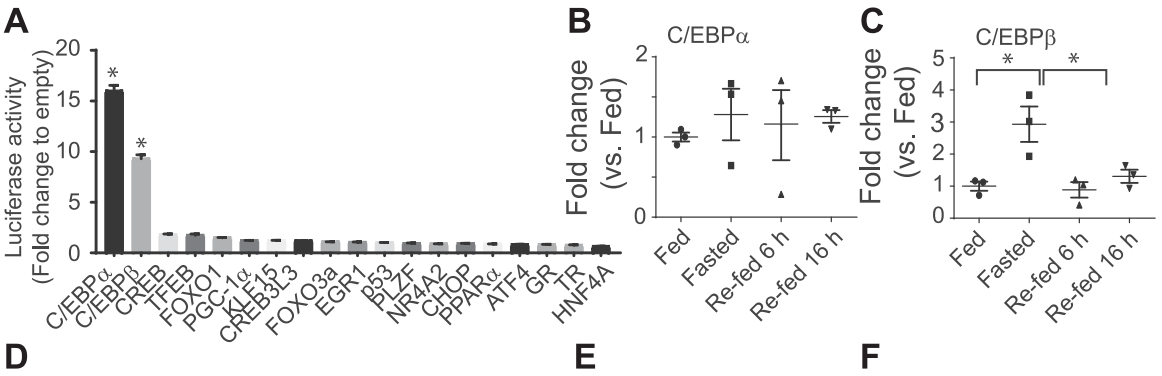

D

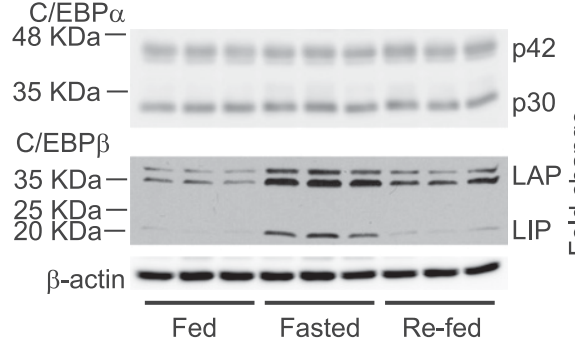

G

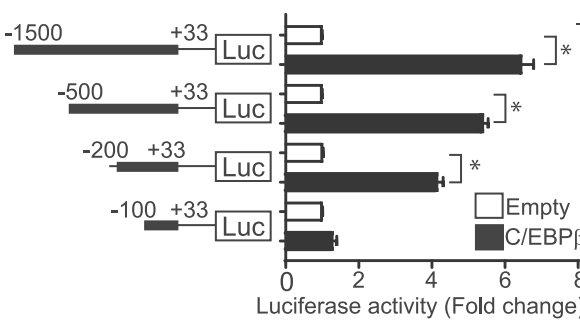

I

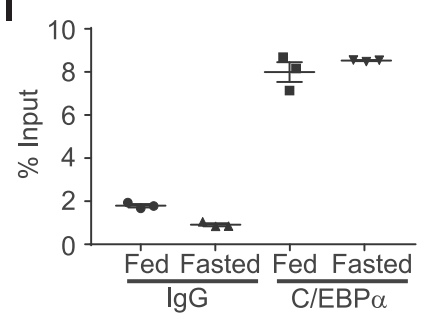

E

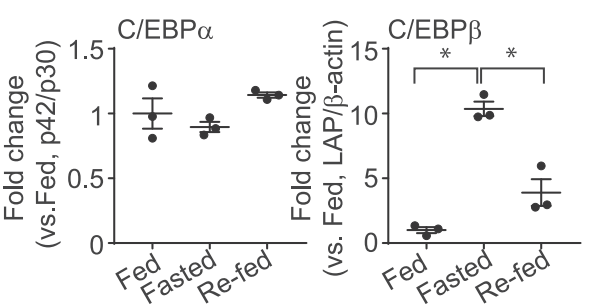

H

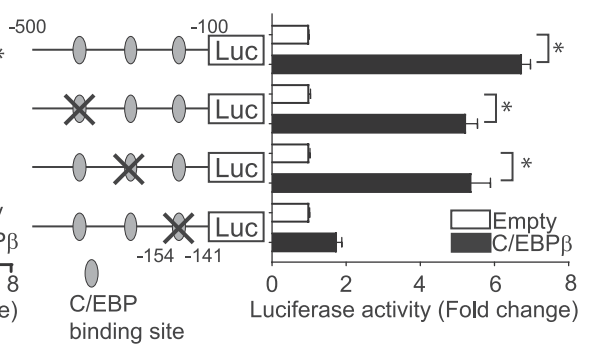

J

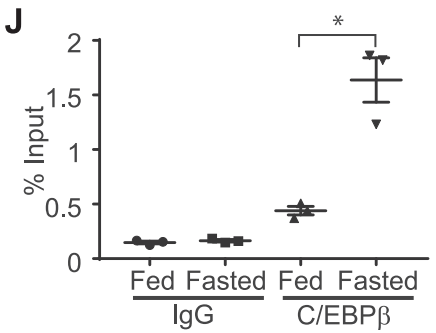

Figure 2. C/EBP $\beta$ mediates FAM134B-2 transcription. (A) Promoter analysis of the mouse FAM134B-2 gene using a luciferase (Luc) reporter gene assay. HEK293T cells were co-transfected with firefly luciferase reporter plasmid containing the 1.5-kb promoter region of the mouse FAM134B-2 gene, PCMV-LacZ, and empty or indicated transcriptional factor expression plasmids. Results are expressed as the relative Luc/galactosidase units of induction ( $n$-fold) over the empty plasmid. (B-D) qRT-PCR analysis and (D) immunoblot analysis of $\mathrm{C} / \mathrm{EBP} \alpha$ and $\mathrm{C} / \mathrm{EBP} \beta$ in mouse livers from fed, fasted, and re-fed conditions. (E, F) Immunoblot results of (E) C/EBP $\alpha$ and (F) C/EBP $\beta$ were quantified using a densitometric analysis. (G, $\mathbf{H})$ Deletion (G) and mutational $(H)$ analysis of the mouse FAM134B-2 gene using a luciferase reporter gene assay. The schematic illustrations represent the serially deleted FAM134B-2/ Luc reporter constructs (G) or mutated FAM134B-2/ Luc reporter constructs $(H)$. Results are expressed as the relative Luc/ $\beta$-galactosidase units of induction ( $n$-fold) over the empty plasmid. (I, J) ChIP assay for in vivo binding status of $C / E B P \alpha(\mathrm{I})$ or $C / E B P \beta(J)$ on the FAM134B-2 promoter. Mouse liver chromatin extracts from fed or fasted conditions were immunoprecipitated with anti-C/EBP antibodies or normal IgG. Purified DNA was determined with qRT-PCR. Data show percentage to input DNA. One-way ANOVA with a Student-Newman post hoc test was used for statistical analysis. ${ }^{*} P<0.05$
stearoyl-CoA desaturase (SCD1) were used as specific markers for SER, whereas ribosome-associated membrane protein-4 (RAMP4) and SEC61 were used as markers for RER. Opti-Prep was able to clearly separate SER and RER (Fig 4A). Interestingly, FAM134B-2 protein is more abundant in SER fractions than RER fractions, whereas FAM134C protein is equally detected in both SER and RER fractions. Another ER-resident cargo receptor, SEC62, is only found in RER fractions (Fig 4A). FAM134A, CCPG1, and RNT3 proteins were not detected in hepatic SER and RER, probably because of very low expression. Immunofluorescence confocal microscopic analysis confirmed that endogenous and exogenous FAM134B-2 is colocalized with both SERCA2 and SEC62 in HeLa cells (Fig S4A-C).

\section{Starvation increases lysosomal FAM134B-2 degradation in vivo and in vitro, and in mice and in humans}

To examine whether starvation increases lysosomal ER protein degradation in vivo, we treated mice with a lysosome inhibitor, leupeptin (i.p., $15 \mathrm{mg} / \mathrm{kg}$ body weight) under fed and fasted states. Leupeptin is most commonly used as an inhibitor for hepatic autophagy (Haspel et al, 2011; Ezaki et al, 2014; Zhang et al, 2015; Moulis \& Vindis, 2017). 4-h leupeptin treatment increased levels of FAM134B-2 protein under the fed state, but increased levels more significantly under the fasted state (Fig 4B and C). As expected, levels of LC3B-II protein were increased by leupeptin treatment, especially under the fasted state (Fig 4B and D). We next examined which ER protein affects starvation-induced ER-phagy. Interestingly, starvation did not change levels of SERCA2, RTN4, and CLIMP63, which are known as FAM134B-1 substrates (Fig 4B and E).

We next examined whether starvation-induced FAM134B and ER-related autophagy can be replicated in primary hepatocytes. However, primary mouse hepatocytes are not a suitable model to study FAM134B-2-mediated ER-related autophagy based on the following observations as shown in Fig S5: 1) Isolation of primary hepatocytes alone induced the expression of FAM134B-2 by more than 50-fold compared with the intact liver (Fig S5A and B). 2) 
A

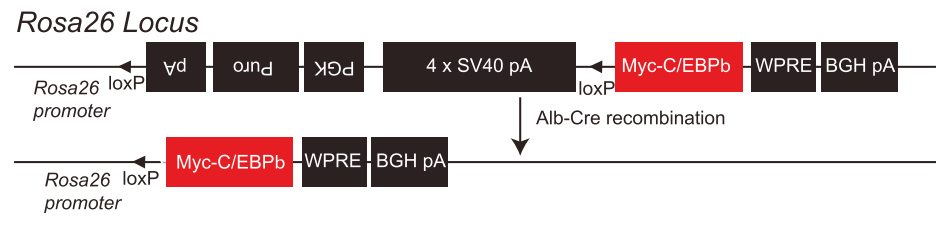

B

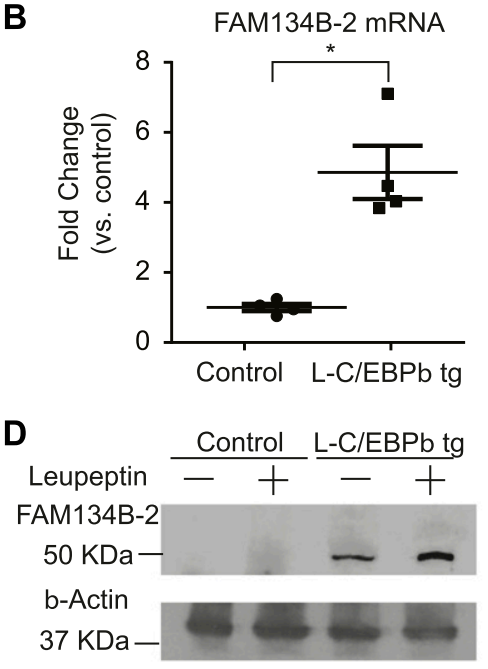

C

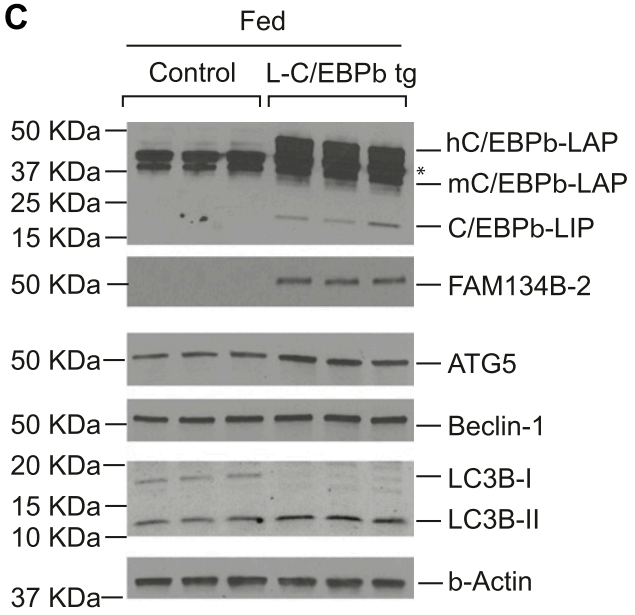

Figure 3. C/EBP $\beta$ regulates FAM134B-2 and autophagy regulators in the mouse liver.

(A) Scheme of construct design for targeting the C/EBP $\beta$ transgene. (B) qRT-PCR analysis of FAM134B-2 in livers from $W T$ and $L-C / E B P \beta$ tg mice under a fed state. (C) Immunoblot analysis of FAM134B-2, ATG5, Beclin-1, and LC3B in livers from WT and L-C/EBP $\beta$ tg mice under a fed state. *Non-specific signal. (D) Immunoblot analysis of FAM134B-2 in livers from WT and L-C/EBP $\beta$ tg mice treated with leupeptin (i.p., $15 \mathrm{mg} / \mathrm{kg}$ body weight) for 4 h. (E) Immunoblot analysis of FAM134B-2 in livers of $\mathrm{L}-\mathrm{C} / \mathrm{EBP} \beta \mathrm{KO}$ mice under starvation. Mice were fasted for 16 h. (F) Quantitative densitometry of the immunoblot analysis. *Nonspecific signal. Student's unpaired $t$ test was used for statistical analysis. ${ }^{*} P<0.05$. Source data are available for this figure.

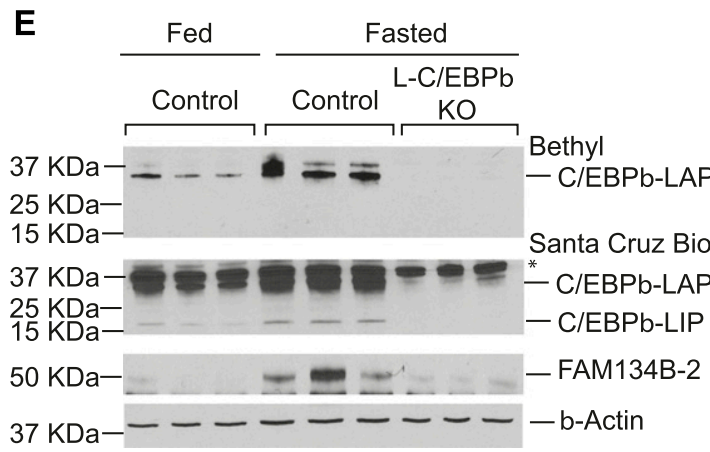

E 
A

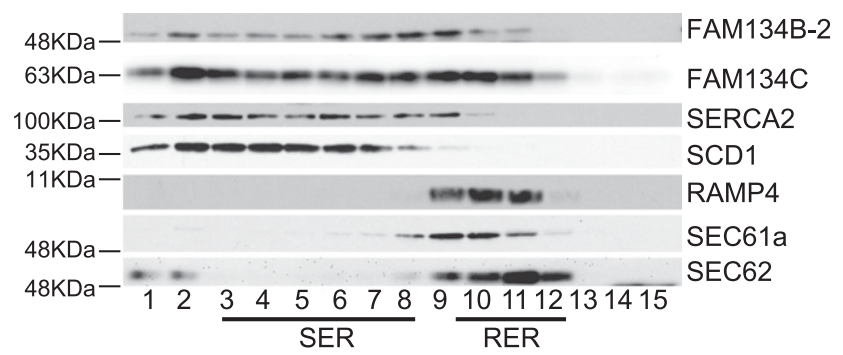

B

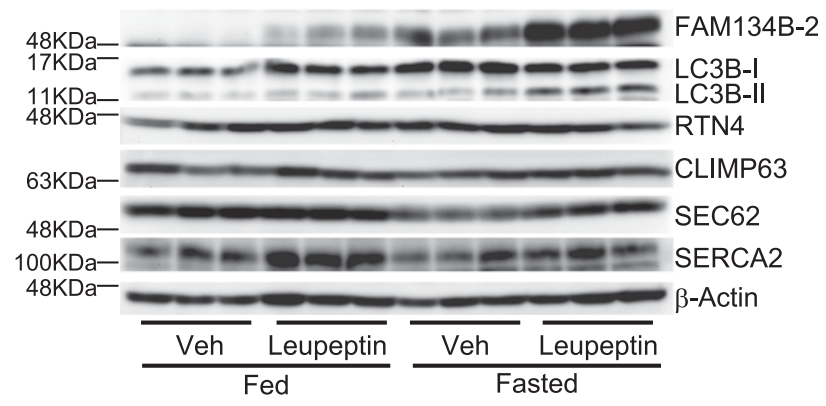

Figure 4. Hepatic FAM134B-2 is located in the SER and RER and degraded in the lysosome.

(A) Immunoblot analysis of FAM134B-2, FAM134C, SERCA2, SCD1, RAMP4, SEC61a, and SEC62 in liver ER subfractions. Microsomes were isolated from fasted mice and separated to ER subfractions using iodixanol density gradient ultracentrifugation (Opti-Prep). (B) Immunoblot analysis of FAM134B-2, LC3B, RTN4, CLIMP63, SEC62, and SERCA2 in the mouse liver. Leupeptin (15 mg/ kg body weight) or PBS was intraperitoneally injected into fed or fasted mice. After $4 \mathrm{~h}$, the mice were sacrificed. (C-E) Densitometric analysis of FAM134B-2 (C), LC3B (D), and SERCA2 (E) in livers with leupeptin treatment. Student's unpaired $t$ test was used for statistical analysis. ${ }^{*} P<0.05$.

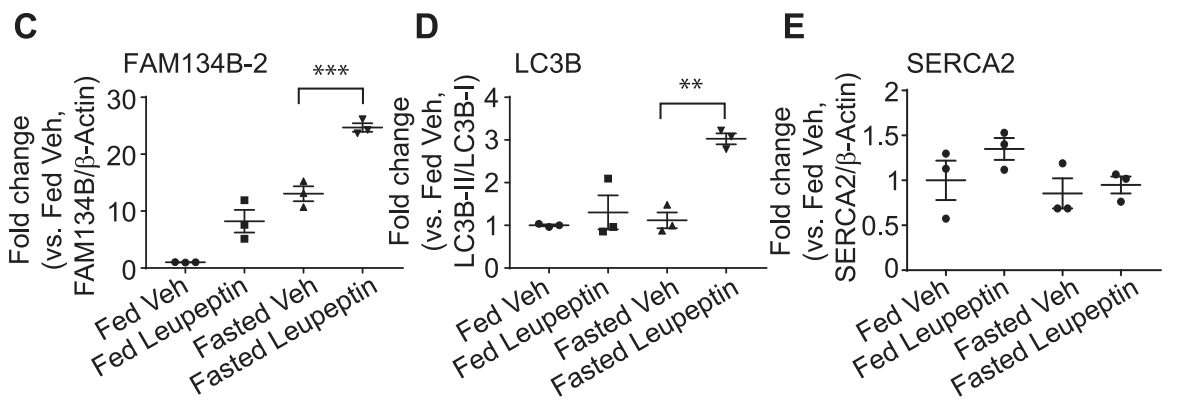

C

coefficient (PCC) analysis, which is widely accepted as a statistical measure of colocalization (McDonald \& Dunn, 2013). PCC confirmed that $\mathrm{C} / \mathrm{EBP} \beta$ overexpression significantly induced FAM134B-2 and LC3 colocalization (Fig 5E). C/EBP $\beta$ overexpression also significantly increased LC3B expression (Fig 5F). Furthermore, this colocalization was observed in LAMP1 positive lysosomes $(23.84 \% \pm 3.03$, $\mathrm{N}=8$, Fig 5G). These results suggest that starvation induces C/EBP $\beta$ FAM134B-2-mediated ER-related autophagy in humans similar to mice.

\section{FAM134B-2 regulates selective degradation of ER-retained secretary proteins but not bulk ER}

Because FAM134B-1 has been identified as an ER-phagy receptor that controls the size of the ER (Khaminets et al, 2015), we next examined whether FAM134B-2 regulates ER size in HeLa cells. Unexpectedly, FAM134B deficiency did not change ER size in HeLa cells (Fig S7A-C). FAM134B-2 reconstitution to FAM134B knockdown cells also did not change ER size. In addition, FAM134B knockdown did not change levels of ER structural proteins and chaperones in HeLa cells (Fig S7D). Furthermore, FAM134B-2 did not change the size of SEC61B-positive sheet-like cisternal ER and RTN4-positive tubular ER (Fig S7E-H). These data suggest that FAM134B-2 does not participate in bulk ER turnover. Recent studies suggest that
FAM134B-1 controls a selective ER-phagy in addition to ER-phagy (Forrester et al, 2018; Schultz et al, 2018). We strived to identify in vivo substrates for FAM134B-2 using FAM134B KO mice (Fig S8A). Since the liver specifically expresses FAM134B-2, ER fractions were isolated from the livers of fasted FAM134B KO and wild-type mice and subjected to a shotgun nontargeted proteomic analysis. The proteomic analysis detected 1,415 proteins and identified 40 proteins that were significantly increased in the microsomes from fasted FAM134B KO mice (Fig 6A) compared with the microsomes from fasted wild-type mice. In this study, we focused on ApoCIII because 1) it was consistently increased second-most in FAM134B KO liver microsomes, 2) it is a secretary protein modified in the ER (Khetarpal et al, 2017), 3) it is very abundant in the liver (Reue et al, 1988), and 4) a specific antibody is commercially available. Immunoblot analysis confirmed that levels of ApoCIII were significantly higher in the microsomal protein fractions from FAM134B KO mice (Figs $6 \mathrm{~B}$ and S8B). mRNA levels of ApoCIII were similar in wildtype and FAM134B KO mice under fasting conditions (Fig S8C). Levels of ER-resident proteins such as Climp63, RTN4, BiP, and calnexin were not changed in the total protein and the microsomal protein fractions of livers from fasted FAM134B KO mice (Figs 6B and S8D). Starvation reduced protein levels of $A p o C I I I$, whereas liver $C / E B P \beta$ deficiency increased levels of ApoCIII under fasting conditions (Figs $6 \mathrm{C}$ and $\mathrm{S} 8 \mathrm{E})$. In contrast, $\mathrm{C} / \mathrm{EBP} \beta$ overexpression in the liver resulted 


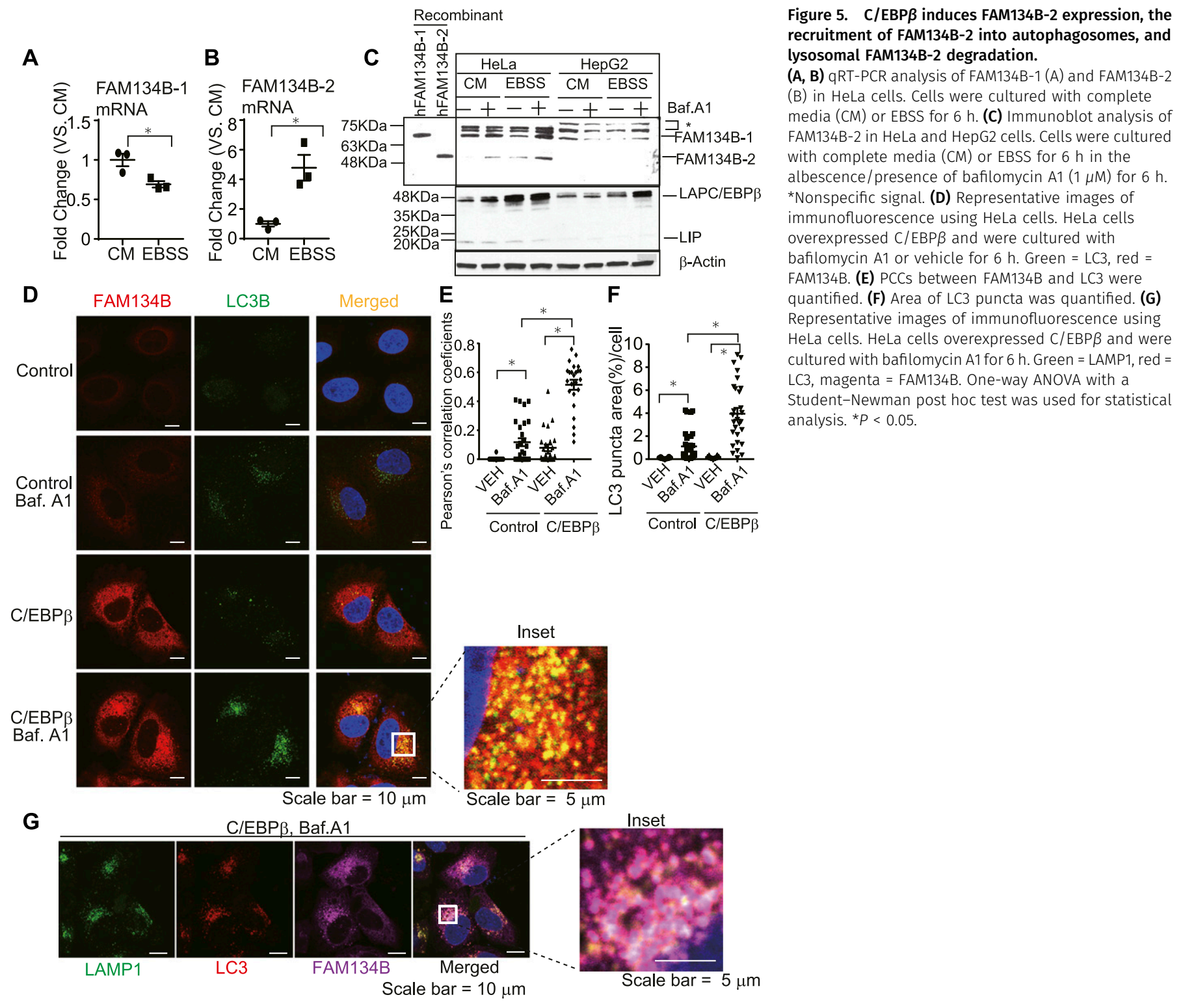

in decreased levels of ApoCIII under fed conditions (Figs 6D and S8F). Immunofluorescence confocal microscopic analysis confirmed that FAM134B deficiency led to ApoCIII accumulation in the ER of HeLa cells (yellow), whereas FAM134B-2 reconstitution normalized ER-retained ApoCIII levels (Fig 7A-C). Coimmunoprecipitation revealed that FAM134B-2 interacted with ApoCIII under EBSS starvation conditions (Fig 7D). To examine whether lysosomal FAM134B-2 and ApoCIII degradation are ATG7dependent, ATG7 KO HeLa cells were generated (Fig S8G). Levels of FAM134B-2 and ApoCIII were significantly increased in ATG7 KO cells (Fig S8G and H).ATG7 deficiency also increased levels of ER-retained ApoCIII and blocked the delivery of ApoCIII to lysosomes (Fig S8I and J). Immunofluorescence confocal microscopy revealed that FAM134B-2 (green) colocalized with FAM134B-2-ApoCIII complexes (yellow) in the lysosome (magenta: ring-like structure) under starved conditions (Fig 7E and F). FAM134B deficiency failed to deliver ApoCIII (red) to the lysosome (green: ring-like structures) and FAM134B-2 reconstitution normalized ApoCIII delivery to the lysosome (Fig $7 \mathrm{G}$ and $\mathrm{H}$ ).

\section{Discussion}

Starvation induces hepatic ER-phagy (Komatsu et al, 2005). Four ERresident cargo receptors (FAM134B, CCPG1, RTN3, and SEC62) have been identified so far (Khaminets et al, 2015; Fumagalli et al, 2016; Grumati et al, 2017; Smith et al, 2018). However, which ER-resident cargo receptor contributes to starvation-induced ER-phagy was not elucidated in vivo. In this study, we have identified a truncated isoform of FAM134B (FAM134B-2) as a starvation-sensitive ERresident cargo receptor. Starvation drastically induced FAM134B-2 but no other ER-resident cargo receptors, including FAM134B-1. 


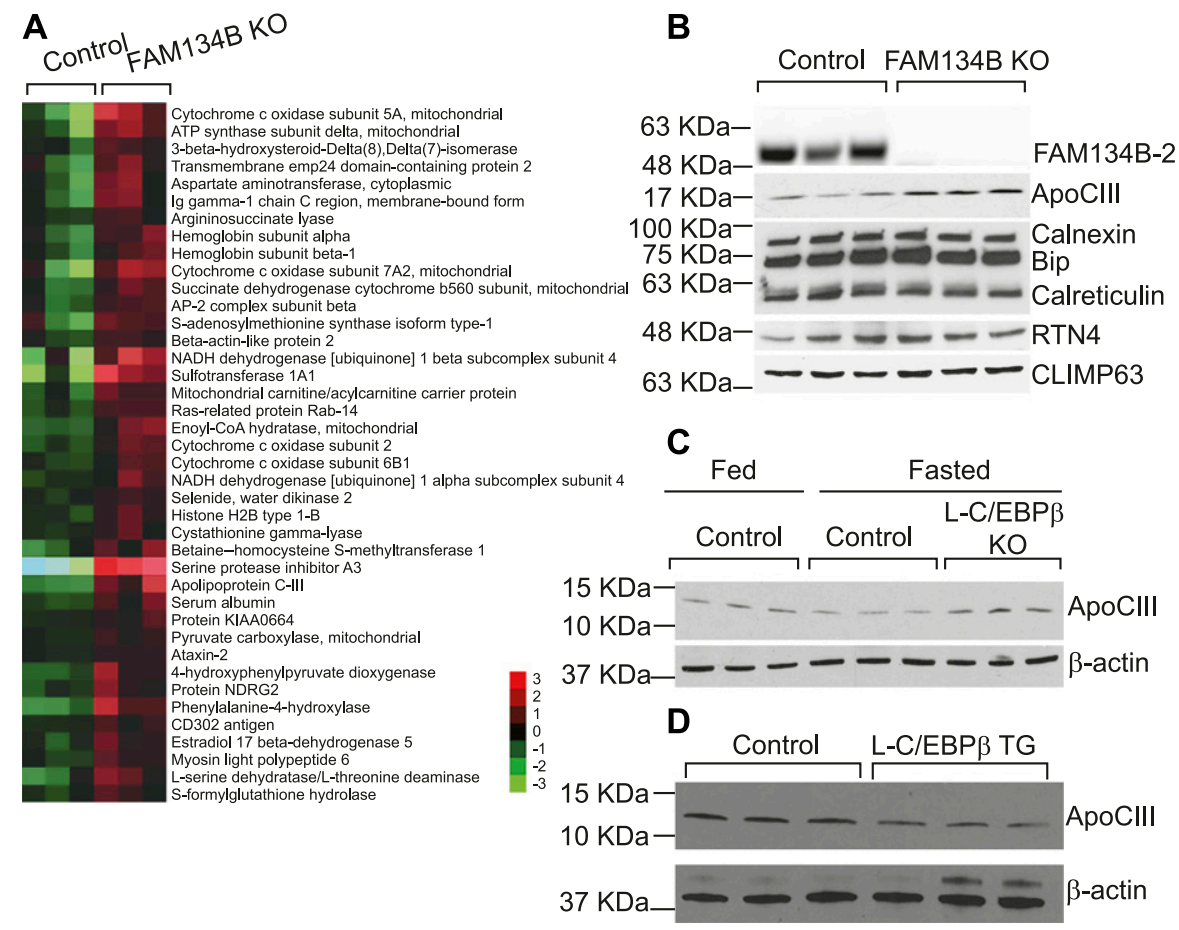

Figure 6. FAM134B-2 induces starvation-induced ApoCIII degradation.

(A) Heat map comparing protein levels in hepatic microsomes of FAM134B KO mice under starvation. Mice were fasted for $16 \mathrm{~h}$. The details of the proteomics analysis were described in the Materials and Methods section. (B) Immunoblot analysis of microsomal ApoCIII, ER chaperones, RTN4, and CLIMP63 in the livers from fasted wild-type and FAM134B KO mice. (C) Immunoblot analysis of ApoCIII in the livers of L-C/EBP $\beta$ KO mice under fasted conditions. (D) Immunoblot analysis of ApoCIII in the livers of L-C/EBP $\beta$ TG mice under fed conditions.

Source data are available for this figure.
The induction of FAM134B-2 by starvation was observed not only in the liver but also in multiple peripheral organs, including the kidneys, spleen, and white adipose tissue. FAM134B-2 is localized in the ER and is capable of interacting with LC3B-II. In addition, the lysosomal degradation of FAM134B-2 was significantly increased under starvation. These results suggest that FAM134B-2 is a major ER-resident cargo receptor contributing to starvation-induced ER-phagy.

$\mathrm{C} / \mathrm{EBP} \beta$ (also called LAP) is a pleiotropic transcription factor that regulates cell differentiation, lipid metabolism, and gluconeogenesis (Rahman et al, 2007, 2016; Xu et al, 2013; Guo et al, 2015). We have identified C/EBP $\beta$ as a key regulator of starvation-induced FAM134B-2 expression and ER-related autophagy. Previously, more than 15 transcription factors were found to be regulators of fastinginduced hepatic gene expression (Goldstein \& Hager, 2015). We tested 19 transcription factors known to control fasting-induced gene expression and autophagy gene expression in the regulation of FAM134B-2 transcription. FAM134B-2 promoter analysis revealed that $C / E B P \alpha$ and $C / E B P \beta$ strongly increased transcriptional activity. Consistently, ATG40, a putative yeast homologue of FAM134B, was induced by a starvation-mimic reagent, rapamycin, which alters C/EBP $\beta$ expression (Begay et al, 2015; Mochida et al, 2015). We also identified a specific element of the FAM134B-2 promoter that drives $C /$ EBP-induced promoter activation. However, because starvation induced hepatic C/EBP $\beta$ but not C/EBP $\alpha$ expression, starvation increased the recruitment of $C / E B P \beta$ but not $C / E B P \alpha$ onto the FAM134B-2 promoter, hepatic C/EBP $\beta$ overexpression strongly induced FAM134B-2 expression under the fed state, and liver $C / E B P \beta$ deficiency significantly blocked starvation-induced FAM134B-2 expression, we think that $C / E B P \beta$ is more critical than $C / E B P \alpha$ in the regulation of starvation-induced hepatic FAM134B-2 expression.
Hepatic C/EBP $\beta$ expression directly induced autophagy-related genes and proteins such as ATG5 and LC3B-II. Furthermore, we were able to replicate starvation-induced FAM134B-2 expression in vitro using a human cell line, HeLa, by treating cells with a starvation media, EBSS, which is commonly used for inducing autophagy. Both in vivo and in vitro, starvation and $C / E B P \beta$ overexpression induced the recruitment of FAM134B-2 into autophagosomes and lysosomal FAM134B-2 degradation. Starvation induced lysosomal FAM134B-2 degradation, which was increased by bafilomycin $A 1$ and leupeptin treatment in vitro and in vivo, respectively. In HeLa cells, C/EBP $\beta$ overexpression remarkably increased not only expression of FAM134B-2 but also the colocalization of FAM134B-2 and LC3. These data suggest that C/EBP $\beta$ regulates FAM134B-2-mediated ER-related autophagy. We need to determine which nutrients and hormones mediate the activation of C/EBP $\beta$ in the regulation of FAM134B-2-mediated ERrelated autophagy. Previous reports showed that FXR, PPAR $\alpha$, and CREB coordinately regulate fasting-induced hepatic autophagy through the transcriptional regulation of autophagy genes including LC3 and ATG5 (Lee et al, 2014; Seok et al, 2014). However, PPAR $\alpha$ and CREB overexpression did not affect FAM134B-2 promoter activity. In addition, treatment of mice with a PPAR $\alpha$ agonist (GW7647) and FXR deficiency did not affect hepatic FAM134B-2 expression, whereas a known PPAR $\alpha$ target, FGF21, was significantly increased by GW7647 (Fig S9A-C).

FAM134B-2 localizes in the SER and RER, whereas another ER-phagy receptor, SEC62, is specifically localized in the RER. These results suggest that whereas SEC62 specifically regulates hepatic RER-related autophagy, FAM134B2 contributes to both SER- and RER-related autophagy. In this study, we were unable to examine whether FAM134B-1 and FAM134B-2 localize in different regions of 
A

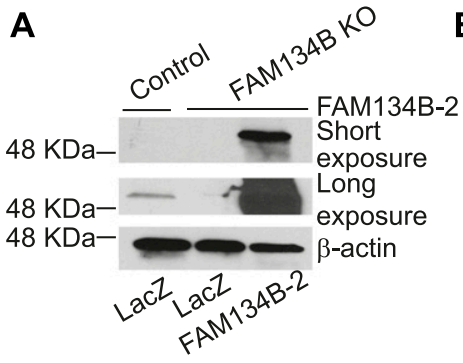

C

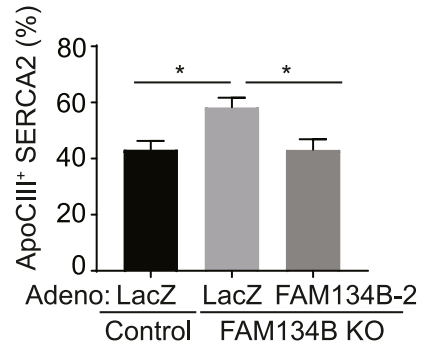

E

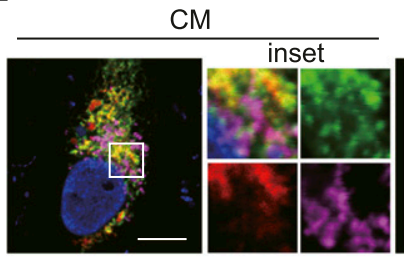

GFP-FAM134B-2 mCherry-ApoCIII LAMP1 Scale bar $=10 \mu \mathrm{m}$

G

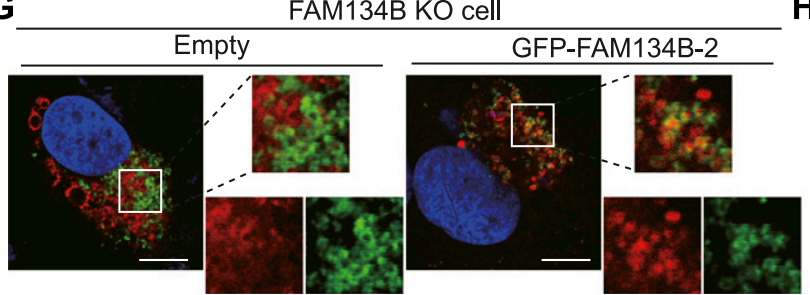

mCherry-ApoCIII LAMP1 Scale bar $=10 \mu \mathrm{m}$
B $\frac{\text { Control cell }}{\text { LacZ }}$

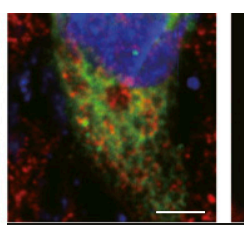

D
FAM134B KO cell

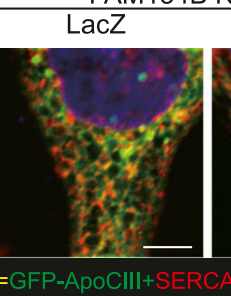

$-2$

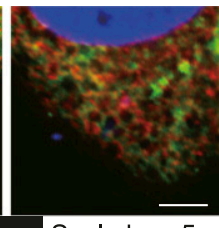

Scale bar $=5 \mu \mathrm{m}$
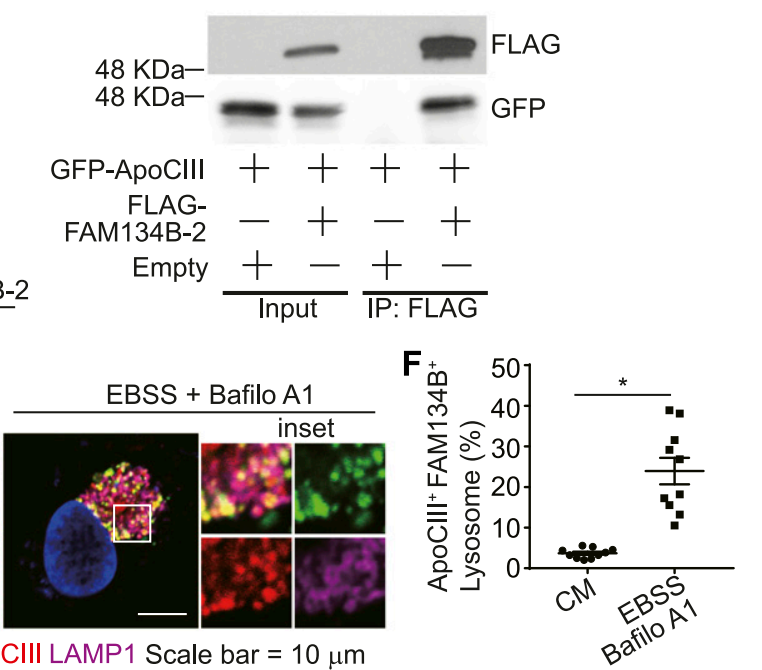

H

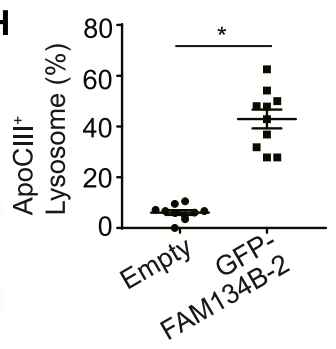

Figure 7. FAM134B-2 induces starvation-induced ApoCIII degradation.

(A-C) Quantification of colocalization of EGFP-ApoCIII and SERCA2 positive ER. FAM134B KO HeLa cells were infected with adenovirus containing mouse FAM134B-2 and transfected with pEGFP-ApoCIII. (A) Immunoblot analysis of FAM134B-2 in HeLa FAM134B KO cells. (B) Representative images of HeLa FAM134B KO cells. (C) Quantification of GFP-ApoCIII positive areas in SERCA2positive ER. (D) Co-immunoprecipitation of endogenous GFP-ApoCIII, FLAG-FAM134B-2. FLAG-FAM134B-2 was transiently overexpressed in HEK293T cells. $24 \mathrm{~h}$ after transfection, the cells were starved for $6 \mathrm{~h}$ in the presence of EBSS and bafilomycin A1. (E, F) Quantification of GFP-FAM134B-2 and mCherry-ApoCIII double positive LAMP1. The cells were transfected with GFP-FAM134B-2 and mCherry-ApoCIII. $24 \mathrm{~h}$ after transfection, the cells were starved for $6 \mathrm{~h}$ in the presence of EBSS and bafilomycin A1 or cultured with complete media. (E) Representative images of HeLa cells. (F) Quantification of GFP-FAM134B-2 and mCherryApoCIII double positive LAMP1. (G, H) Quantification of mCherry-ApoCIII-positive LAMP1. FAM134B KO HeLa cells were transfected with GFP-FAM134B-2 and mCherryApoCIII. $24 \mathrm{~h}$ after transfection, the cells were starved for $6 \mathrm{~h}$ in the presence of EBSS and bafilomycin A1. (G) Representative images of HeLa cells. (H) Quantification of mCherry-ApoCIII-positive LAMP1. One-way ANOVA with a Student-Newman post hoc test was used for statistical analysis. ${ }^{*} P<0.05$ the ER or examine the subcellular localization of other ER-resident cargo receptors such as full-length RTN3 and CCPG1 because of the lack of hepatic expression.

Although FAM134B-1 was originally identified as an ER-phagy receptor, recent studies suggest that FAM134B-1 regulates selective ER-phagies such as the degradation of protein procollagens, a native secretary protein family, which were identified as endogenous substrates for FAM134B-1 in fibroblasts (Forrester et al, 2018) and a Niemann-Pick C1 mutant (NPC1-I1061T) synthesized and modified in the ER (Schultz et al, 2018). We performed the functional analysis of FAM134B-2 to test whether FAM134B-2 regulates ER-phagy. FAM134B deficiency and FAM134B-2 reconstitution do not change the size of the ER and/or ER structure proteins in HeLa cells and mouse livers, suggesting that FAM134B-2 does not participate in bulk ER turnover. We next examined whether FAM134B-2 regulates selective ER protein degradation using a nontargeted proteomics approach in the hepatic microsome (crude ER) fraction of FAM134B KO mice. Interestingly, none of the ER structure proteins or ER chaperones were changed in the livers of FAM134B KO mice. Most of the identified proteins are either secretary proteins (i.e., ApoCIII, SERPINA3, albumin, and hemoglobins) or mitochondrial proteins (i.e., cytochrome oxidases and ATP synthase). The ER is a critical site for the secretory protein system (Benham, 2012). ApoCIII is a component of triglyceride-rich lipoproteins such as VLDL (Khetarpal et al, 2017). In this study, we identified ApoCIII as one of the hepatic substrates for FAM134B-2mediated selective ER-phagy. Co-immunoprecipitation showed that FAM134B-2 physically interacted with ApoCIII. Starvation reduced levels of ER-retained ApoCIII in an ATG7-dependent manner and increased the delivery of FAM134B-2-ApoCIII complexes to lysosomes. FAM134B deficiency failed to deliver ApoCIII to lysosomes, resulting in a significant accumulation of $A p o C I I I$ in the $E R$, whereas FAM134B-2 reconstitution to FAM134B KO cells normalized the delivery of ApoCIII to lysosomes. In addition, hepatic C/EBP $\beta$ deficiency blocked starvation-induced FAM134B-2, leading to the accumulation of ApoCIII, whereas C/EBP $\beta$ overexpression reduced levels of hepatic ApoCIII. 
Previous studies exclusively examined the role of FAM134B-1 in the regulation of ER-phagy, colon cancer, vascular dementia, viral infection, and neuropathy (Kurth et al, 2009; Kong et al, 2011; Kasem et al, 2014; Khaminets et al, 2015; Islam et al, 2018). FAM134B-1 expression is limited in the brain, spleen, and testis. Although many of the peripheral tissues express both FAM134B-1 and FAM134B-2 isoforms at very low levels under normal conditions, starvation drastically induces FAM134B-2 but not FAM134B-1 in the peripheral tissues through the transcriptional activation of $C / E B P \beta$. Further studies are required for examining why two isoforms of FAM134B in addition to three other ER-resident LC3B-II-binding proteins are present in the mammalian ER and the physiological role of the C/EBP $\beta$-FAM134B-2-ER-lysosome protein degradation pathway. We believe that the present study sheds light on novel insights into starvation-induced selective ER-phagy.

\section{Materials and Methods}

\section{Animals}

To generate liver-specific C/EBP $\beta$ TG mice, we introduced the transgene at the Rosa26 locus by the recombinase-mediated cassette exchange method that we recently developed to circumvent the inherent problem of random insertion via traditional pronuclear injection (Masuda et al, 2016; Shiozaki et al, 2018). In brief, we transfected $5 \times 10^{6}$ R26FNF3-1F1 ES cells with $15 \mu \mathrm{g}$ of pFLSLF3-Myc human C/EBP $\beta$ and $15 \mu \mathrm{g}$ of pCAG-Flpe (plasmid 13787; Addgene). From the 96 clones tested, we found three clones with the expected genotype, which were also G418 sensitive, indicating successful exchange of the original neo cassette for the transgene cassette at the Rosa26 locus. Karyotypically normal ES clones were microinjected into C57BL/ 6 blastocysts to produce chimeric founders at the University of Colorado Bioengineering Core Facility. The generated mice were named Rosa26-C/EBP $\beta$ conditional TG (Rosa26-C/EBP $\beta^{\text {loxtg/+}}$ ) mice. All mouse strains were backcrossed more than 10 times with C57BI/6J mice. The genetic backgrounds were checked at the BioResources Core Facility of the Barbara Davis Center at the University of Colorado-Denver. The Rosa26-C/EBP $\beta^{\text {loxtg/+ }}$ mice were intercrossed with Albumin (Alb)-Cre mice to obtain Alb-Cre; C/EBP $\beta^{\text {loxtg/+ }}$ mice and control Alb-Cre mice. To generate liver-specific $C / E B P \beta$ KO mice, $C / E B P \beta$-floxed mice were purchased from Mutant Mouse Resource and Research Center (MMRRC, 034760-UNC) (Sterneck et al, 2006). The $C / E B P \beta$-floxed mice were intercrossed with Alb-Cre mice to obtain Alb-Cre(+); $C / E B P \beta^{\text {flox/flox }}$ mice and control Alb-Cre(-); $C / E B P \beta^{\text {flox/flox }}$ mice. To generate FAM134B knockout mice, we flanked the last exon of FAM134B by two loxP sites (Fig S6D). In brief, a 2,487bp DNA fragment encompassing the last exon of FAM134B was amplified by PCR using the BAC clone RP23-475N20 as a template. This DNA fragment was then cloned in between two loxP sites in the cloning vector pLFNFL at the Pacl site immediately upstream of an FRT-PGK-neo-polyA-FRT selection cassette to produce PL9FNFL. A left homologous arm encompassing 3,386 bp of DNA sequence upstream of exon 9 was PCR-amplified and cloned at the Clal site, upstream of the proximal loxP site in PL9FNFL. Subsequently, a right homologous arm containing 2,703 bp of DNA sequence downstream of exon 6 was
PCR amplified and cloned at the Notl site, downstream of the distal loxP site. The resulting targeting construct was designated as pFam134b-LFNFL (13,535 bp). The targeting vector was linearized and introduced by electroporation into murine B6/129 hybrid EC7.1 embryonic stem cells. Karyotypically normal ES clones were microinjected into C57BL/ 6 blastocysts to produce chimeric founders at the Bioengineering Core facility in the University of Colorado-Denver. To generate FAM134B KO mice, the FAM134B lox mice were crossed with Rosa-Flp and Ella-Cre mice and backcrossed 10 times with C57Bl6 mice (Fig S7A). 8-wk-old male C57BL6/J (WT) mice were purchased from the Jackson Laboratory. For the fasting and re-feeding study, WT mice were randomly separated to fed and fasted groups. After $16 \mathrm{~h}$ of fasting, mice were intraperitoneally injected with either vehicle or leupeptin (15 mg/kg body weight) (Gold Biotechnology) (Ezaki et al, 2014). Some of the fasted mice were re-fed a chow diet for an additional 6 or $16 \mathrm{~h}$ after fasting. Animal experiments were approved by the Institutional Animal Care and Research Advisory Committee of the University of Colorado at Denver.

\section{Cell cultures}

HEK293T cells (ATCC), C2C12 cells (ATCC), HepG2 cells (ATCC), and HeLa cells (ATCC) were grown and maintained at $37^{\circ} \mathrm{C}$ in an atmosphere of $5 \% \mathrm{CO}_{2}$ in DMEM containing 4,500 mg/l glucose, $100 \mathrm{U} /$ $\mathrm{ml}$ penicillin, and $100 \mu \mathrm{g} / \mathrm{ml}$ streptomycin and supplemented with $10 \%$ FBS. Mouse primary hepatocytes were isolated as described previously (Nakatani et al, 2002) and cultured in DMEM containing $1,000 \mathrm{mg} / \mathrm{l}$ glucose, $100 \mathrm{U} / \mathrm{ml}$ penicillin, and $100 \mu \mathrm{g} / \mathrm{ml}$ streptomycin and supplemented with 10\% FBS. For starvation experiments, the cells were starved with EBSS and treated with $1 \mu \mathrm{M}$ bafilomycin A1 or vehicle for 6 or $8 \mathrm{~h}$.

\section{5'RACE}

5'RACE was performed using the SMARTer RACE 5'/3' kit (Takara) according to the manufacturer's instructions. cDNA was prepared with the SMARTer RACE 5' / $3^{\prime}$ Kit using $1 \mu \mathrm{g}$ of RNA from mouse liver or brain. CDNA was amplified with mouse FAM134B-specific reverse primers, shown in Table S1. 5'RACE products were cloned into pGEM-T Easy vector (Promega) and sequenced.

\section{RNA analysis}

qRT-PCR assays were performed using an Applied Biosystems StepOne Plus qPCR instrument. Quantitative expression values were calculated by the absolute standard curve method using plasmid template (Open Biosystems) containing each target gene cDNA. Primer sequences are listed in Table S1.

\section{Protter}

Amino acid sequences of FAM134B-2 (NP_001264244.1) were obtained from National Center for Biotechnology Information. Transmembrane topology of FAM134B-2 was predicted and visualized using Protter ver.1.0 (Omasits et al, 2014). 


\section{Transfections and luciferase assays}

HEK293T cells were plated in 24-well plates and grown overnight. The cells were transfected with $300 \mathrm{ng}$ firefly luciferase reporter plasmids containing 1.5-kb mouse FAM134B-2 promoter (pGL3; Promega), 300 ng expression plasmid, and 50 ng of pCMV-LacZ vector using Turbofect (Thermo Fisher Scientific). After $24 \mathrm{~h}$ of transfection, the cells were harvested with $100 \mu \mathrm{l}$ passive lysis buffer, and luciferase activities were measured using a luciferase assay system (Promega). Firefly luciferase activity was divided by $\beta$-galactosidase activity to obtain a normalized value, the relative luciferase unit.

\section{ChIP analysis}

The samples were prepared using SimpleChIP Enzymatic Chromatin IP Kit (Magnetic Beads) from Cell Signaling Technology. ChIP was performed using rabbit IgG control C/EBP $\alpha$ or C/EBP $\beta$ antibody. Eluted DNA was analyzed by qRT-PCR. The results are expressed as the percentage of antibody binding versus the amount of PCR product obtained using a standardized aliquot of input chromatin (\% of input). The primers for the C/EBP composite region of the mouse FAM134B-2 promoter are shown in Table S1.

\section{ER fractionation}

ER fractionation was performed using an ER isolation kit (ER0100; Sigma-Aldrich) according to the manufacturer's instructions. Microsomes isolated from 16-h fasted mouse livers were subjected to 15-30\% Opti-Prep gradient and centrifuged at 150,000 $\mathrm{g}$ for $3 \mathrm{~h}$ at $4^{\circ} \mathrm{C}$. After centrifugation, 15 fractions were collected from the top to the bottom of the gradient and subjected to immunoblot analysis.

\section{Immunoblot analysis}

Cell and tissue lysates were prepared using RIPA buffer (150 mM $\mathrm{NaCl}, 1 \%$ Nonidet P-40, $0.5 \%$ sodium deoxycholate, $0.1 \%$ SDS, and 50 $\mathrm{mM}$ Tris, $\mathrm{pH}$ 8.0). The samples were separated by SDS-PAGE, transferred to a nitrocellulose membrane, and immunoblotted with the primary antibody. The samples were visualized using horseradish peroxidase coupled to appropriate secondary antibodies with enhancement by an ECL detection kit (Thermo Fisher Scientific). Primary antibodies are listed in Table S2. The FAM134B rabbit polyclonal antibody was produced against a peptide ((C) LPTELKRKKQQLDSAHR, amino acids 352-368; NP_001030023.1). The specificity of the FAM134B antibody was tested with immunoblot analysis using protein lysates from primary hepatocytes and $\mathrm{C}_{2} \mathrm{C} 12$ myoblasts treated with FAM134B siRNA (s82925; Thermo Fisher Scientific) as shown in Fig 510 . The antibody recognized both mouse FAM134B-1 and FAM134B-2.

\section{Immunofluorescence}

HeLa cells were grown on chamber slides, and after each experiment, the cells were fixed with $3 \%$ paraformaldehyde for $15 \mathrm{~min}$. The cells were permeabilized with $0.05 \%$ digitonin in PBS for $10 \mathrm{~min}$ and blocked with $0.1 \%$ gelatin in PBS for $30 \mathrm{~min}$. Subsequently, the cells were incubated with primary antibody solution for $1 \mathrm{~h}$, washed in the permeabilizing buffer, and then incubated with secondary antibody solution for $1 \mathrm{~h}$. Coverslips were washed three times and mounted on microscope slides using Vectashield mounting medium with DAPI (Vector Laboratory). Fluorescence signals were captured using an Olympus FV1000 FCS/RICS (Olympus). To quantify the colocalization of FAM134B and LC3B, PCCS from at least 20 cells were calculated using NIH ImageJ. Primary antibodies are listed in Table S2.

\section{ER size quantitation}

HeLa cells stably expressing mCherry-ER, mCherry-SEC61B, and HA-RTN4 were infected with adenovirus containing mouse FAM134B-2 and then treated with FAM134B siRNA (s29014; Thermo Fisher Scientific). Adenovirus containing mouse FAM134B-2 and lentivirus containing ER-mCherry (\#55041; Addgene) were generated using the Gateway cloning system (Invitrogen). ER area measurements (20 cells) were conducted using NIH ImageJ as follows. Background threshold was manually defined and set for all images. Borders of each cell were drawn and the ER area was then calculated and presented as an ER-fraction of the total cell area.

\section{ER ApoCIII accumulation}

HeLa cells lacking FAM134B or ATG7 were generated by the CRISPRCas9 system as previously described (Shiozaki et al, 2018). The sgRNA target sequences are shown in Table S1. Wild-type and FAM134B KO HeLa cells expressing GFP-ApoCIII were infected with adenovirus containing mouse FAM134B-2 or LacZ (control). Lentivirus containing GFP-ApoCIII was generated using the Gateway cloning system (Invitrogen). The cells were immunolabeled with anti-SERCA2 antibody as described in the immunofluorescence section. Fluorescence signals were captured using an Olympus FV1000 FCS/RICS (Olympus). GFP-ApoCIII-positive SERCA2 areas from 20 cells were calculated using NIH ImageJ. Background threshold was manually defined and set for all images.

\section{Co-immunoprecipitation}

FLAG-mouse FAM134B-2, FAM134B2- $\triangle$ LIR, and GFP-ApoCIII overexpressed HEK293T cells were starved with EBSS for $6 \mathrm{~h}$ in the presence of bafilomycin $\mathrm{A} 1(1 \mu \mathrm{M})$. Cell lysates were prepared using co-immunoprecipitation buffer $(150 \mathrm{mM} \mathrm{NaCl}, 1 \%$ Nonidet P-40, and $50 \mathrm{mM}$ Tris, pH 8.0). $2 \mathrm{mg}$ of lysate and $40 \mu \mathrm{l}$ of anti-DYKDDDDK G1 Affinity Resin (GenScript) were incubated overnight at $4^{\circ} \mathrm{C}$. Resins were washed three times with co-immunoprecipitation buffer. Immunoprecipitated proteins were analyzed using immunoblot. The plasmid containing FAM134B2- $\Delta$ LIR was generated by replacing the LIR sequence (DDFELL) with alanines using a Q5 Site-Directed Mutagenesis Kit (New England Biolabs).

\section{Nontargeted proteomics}

Hepatic microsome fractions $(\mathrm{N}=3)$ were isolated using sequential ultracentrifugation as previously described (Man et al, 2006; Masuda et al, 2015). Proteomics was performed at the University of Colorado Mass Spectrometry Core Facility. The samples were digested using a modified filter-aided sample preparation method. Briefly, the samples were denatured, reduced, and alkylated using 4\% SDS in $100 \mathrm{mM}$ Tris- $\mathrm{HCl}$ buffer at $\mathrm{pH}$ 8.5, tris(2-carboxyethyl)phosphine (TCEP), and 
iodoacetamide, respectively. SDS was removed using an Amicon Ultra 30-kD molecular weight cutoff spin filter by sequentially washing with $100 \mathrm{mM}$ Tris- $\mathrm{HCl}$, pH 8.5, containing $8 \mathrm{M}$ urea followed by $2 \mathrm{M}$ urea. The protein was then digested at room temperature for $4 \mathrm{~h}$ with a ratio of 1:50 Lys-C (Wako chemicals) to substrate and then overnight at $37^{\circ} \mathrm{C}$ with a ratio of 1:40 trypsin to substrate. The resulting peptide solutions were recovered by centrifugation at 14,000 $\mathrm{g}$ for $10 \mathrm{~min}$ and washed twice with $80 \mu \mathrm{L}$ of $0.5 \mathrm{M} \mathrm{NaCl}$ solution. The samples were desalted using Pierce C18 spin columns, according to the manufacturer's instructions. Desalted peptide samples were then evaporated to dryness in a Speed Vac concentrator at $45^{\circ} \mathrm{C}$. The dried samples were resuspended in 3\% ACN, $0.1 \%$ formic acid in water for LC-MS/MS analysis. Pooled samples were loaded into a $2 \mathrm{~cm}$ PepMap 100, nanoviper trapping column and chromatographically resolved online using a $0.075 \times 250$ mm, $2.0 \mu \mathrm{m}$ Acclaim PepMap RSLC reversephase nanocolumn (Thermo Fisher Scientific) using a 1,290 Infinity II LC system equipped with a nanoadapter (Agilent). Mobile phases consisted of water $+0.1 \%$ formic acid $(A)$ and $90 \%$ aq. acetonitrile + $0.1 \%$ formic acid (B). The samples were loaded into the trapping column at $3.2 \mu \mathrm{l} / \mathrm{min}$ for $2.5 \mathrm{~min}$ at an initial condition before being chromatographically separated at an effective flow rate of $330 \mathrm{nl} / \mathrm{min}$ using a gradient of 3-8\% B over 4 min, 8-27\% B over $101 \mathrm{~min}$, and $27-40 \%$ B over $15 \mathrm{~min}$ for a 120 -min total gradient at $42^{\circ} \mathrm{C}$. The gradient method was followed by a column wash at $70 \%$ B for 5 min. Data were collected on a 6550 Q-TOF equipped with a nano-source (Agilent) operated using intensity-dependent CID MS/MS to generate peptide identifications. The capillary voltage, drying gas flow, and drying gas temperature were set to $1,300 \mathrm{~V}, 11.0$ liters $/ \mathrm{min}$, and $175^{\circ} \mathrm{C}$, respectively. MS/MS data were collected in positive ion polarity over mass ranges $290-1,700 \mathrm{~m} / \mathrm{z}$ at a scan rate of 10 spectra/ $\mathrm{s}$ for MS scans and mass ranges 50-1,700 $\mathrm{m} / \mathrm{z}$ at a scan rate of 3 spectra/s for MS/MS scans. All charge states, except singly charged species, were allowed during MS/MS acquisition, and charge states 2 and 3 were given preference. SpectrumMill software (Agilent) was used to extract, search, and summarize peptide identity results. Spectra were searched against the SwissProt Mus Musculus database, allowing up to two missed tryptic cleavages with fixed carbamidomethyl (C) and variable deamidated $(\mathrm{N})$, oxidation $(\mathrm{M})$, and pyroglutamic acid (Q) modifications. The monoisotopic peptide mass tolerance allowed was $\pm 20.0 \mathrm{ppm}$ and the MS/MS tolerance was $\pm 50 \mathrm{ppm}$. A minimum peptide score of 8 and scored peak intensity of $50 \%$ were used for generation of the AMRT library. The entire proteomics data are shown in Table S3.

\section{Statistics}

Data were collected from more than two independent experiments and are reported as the mean \pm SEM. Statistical analysis was performed using a two-tailed $t$ test for two-group comparison and a oneway ANOVA with a Student-Newman post hoc test or two-way ANOVA for multigroup comparison. Significance was accepted at $P<0.05$.

\section{Supplementary Information}

Supplementary Information is available at https://doi.org/10.26508/lsa. 201900340.

\section{Acknowledgements}

The authors' work was supported by grants from National Institutes of Health R01DK096030, R01HL117062, R01HL133545, and R01HL132318 to M. Miyazaki.

\section{Author Contributions}

S Kohno: conceptualization, data curation, software, formal analysis, validation, investigation, visualization, methodology, and writing-original draft.

Y Shiozaki: conceptualization, resources, and software.

AL Keenan: data curation, formal analysis, investigation, methodology, and writing-original draft, review, and editing.

S Miyazaki-Anzai: resources, data curation, formal analysis, investigation, visualization, and methodology.

M Miyazaki: conceptualization, resources, data curation, software, formal analysis, supervision, funding acquisition, validation, investigation, visualization, methodology, project administration, and writing-original draft, review, and editing.

\section{Conflict of Interest Statement}

The authors declare that they have no conflict of interest.

\section{References}

Begay V, Smink JJ, Loddenkemper C, Zimmermann K, Rudolph C, Scheller M, Steinemann D, Leser U, Schlegelberger B, Stein H, et al (2015) Deregulation of the endogenous C/EBPbeta LIP isoform predisposes to tumorigenesis. J Mol Med (Berl) 93: 39-49. doi:10.1007/s00109-014$1215-5$

Benham AM (2012) Protein secretion and the endoplasmic reticulum. Cold Spring Harb Perspect Biol 4: a012872. doi:10.1101/cshperspect.a012872

Borgese N, Francolini M, Snapp E (2006) Endoplasmic reticulum architecture: Structures in flux. Curr Opin Cell Biol 18: 358-364. doi:10.1016/ j.ceb.2006.06.008

Braakman I, Hebert DN (2013) Protein folding in the endoplasmic reticulum. Cold Spring Harb Perspect Biol 5: a013201. doi:10.1101/ cshperspect.a013201

Ezaki J, Matsumoto N, Takeda-Ezaki M, Komatsu M, Takahashi K, Hiraoka Y, Taka H, Fujimura T, Takehana K, Yoshida M, et al (2014) Liver autophagy contributes to the maintenance of blood glucose and amino acid levels. Autophagy 7: 727-736. doi:10.4161/auto.7.7.15371

Forrester A, De Leonibus C, Grumati P, Fasana E, Piemontese M, Staiano L, Fregno I, Raimondi A, Marazza A, Bruno G, et al (2018) A selective ERphagy exerts procollagen quality control via a Calnexin-FAM134B complex. EMBO / 38: e99847. doi:10.15252/embj.201899847

Fregno I, Molinari M (2018) Endoplasmic reticulum turnover: ER-phagy and other flavors in selective and non-selective ER clearance. F1000Res 7: 454. doi:10.12688/f1000research.13968.1

Fullgrabe J, Ghislat G, Cho DH, Rubinsztein DC (2016) Transcriptional regulation of mammalian autophagy at a glance. J Cell Sci 129 . 3059-3066. doi:10.1242/jcs.188920

Fumagalli F, Noack J, Bergmann TJ, Cebollero E, Pisoni GB, Fasana E, Fregno I, Galli C, Loi M, Solda T, et al (2016) Translocon component Sec62 acts in endoplasmic reticulum turnover during stress recovery. Nat Cell Biol 18: 1173-1184. doi:10.1038/ncb3423 
Goldstein I, Hager GL (2015) Transcriptional and chromatin regulation during fasting: The genomic era. Trends Endocrinol Metab 26: 699-710. doi:10.1016/j.tem.2015.09.005

Grumati P, Morozzi G, Holper S, Mari M, Harwardt MI, Yan R, Muller S, Reggiori F, Heilemann M, Dikic I (2017) Full length RTN3 regulates turnover of tubular endoplasmic reticulum via selective autophagy. Elife 6. doi:10.7554/elife.25555

Guo L, Li X, Tang QQ (2015) Transcriptional regulation of adipocyte differentiation: A central role for CCAAT/enhancer-binding protein (C/ EBP) beta. J Biol Chem 290: 755-761. doi:10.1074/jbc.r114.619957

Haspel J, Shaik RS, Ifedigbo E, Nakahira K, Dolinay T, Englert JA, Choi AM (2011) Characterization of macroautophagic flux in vivo using a leupeptinbased assay. Autophagy 7: 629-642. doi:10.4161/auto.7.6.15100

Hwang J, Qi L (2018) Quality control in the endoplasmic reticulum: Crosstalk between ERAD and UPR pathways. Trends Biochem Sci 43: 593-605. doi:10.1016/j.tibs.2018.06.005

Islam F, Chaousis S, Wahab R, Gopalan V, Lam AK (2018) Protein interactions of FAM134B with EB1 and APC/beta-catenin in vitro in colon carcinoma. Mol Carcinog 57: 1480-1491. doi:10.1002/mc.22871

Kasem K, Sullivan E, Gopalan V, Salajegheh A, Smith RA, Lam AK (2014) JK1 (FAM134B) represses cell migration in colon cancer: A functional study of a novel gene. Exp Mol Pathol 97: 99-104. doi:10.1016/ j.yexmp.2014.06.002

Khaminets A, Heinrich T, Mari M, Grumati P, Huebner AK, Akutsu M, Liebmann L, Stolz A, Nietzsche S, Koch N, et al (2015) Regulation of endoplasmic reticulum turnover by selective autophagy. Nature 522: 354-358. doi:10.1038/nature14498

Khetarpal SA, Zeng X, Millar JS, Vitali C, Somasundara AVH, Zanoni P, Landro JA, Barucci N, Zavadoski WJ, Sun Z, et al (2017) A human APOC3 missense variant and monoclonal antibody accelerate apoC-III clearance and lower triglyceride-rich lipoprotein levels. Nat Med 23: 1086-1094. doi:10.1038/nm.4390

Komatsu M, Waguri S, Ueno T, Iwata J, Murata S, Tanida I, Ezaki J, Mizushima N, Ohsumi Y, Uchiyama Y, et al (2005) Impairment of starvation-induced and constitutive autophagy in Atg7-deficient mice. J Cell Biol 169: 425-434. doi:10.1083/jcb.200412022

Kong M, Kim Y, Lee C (2011) A strong synergistic epistasis between FAM134B and TNFRSF19 on the susceptibility to vascular dementia. Psychiatr Genet 21: 37-41. doi:10.1097/ypg.0b013e3283413496

Krebs J, Agellon LB, Michalak M (2015) Ca(2+) homeostasis and endoplasmic reticulum (ER) stress: An integrated view of calcium signaling. Biochem Biophys Res Commun 460: 114-121. doi:10.1016/ j.bbrc.2015.02.004

Kroeger H, Chiang WC, Felden J, Nguyen A, Lin JH (2018) ER stress and unfolded protein response in ocular health and disease. FEBS / 286: 399-412. doi:10.1111/febs.14522

Kurth I, Pamminger T, Hennings JC, Soehendra D, Huebner AK, Rotthier A, Baets J, Senderek J, Topaloglu H, Farrell SA, et al (2009) Mutations in FAM134B, encoding a newly identified Golgi protein, cause severe sensory and autonomic neuropathy. Nat Genet 41: 1179-1181. doi:10.1038/ng.464

Lee JM, Wagner M, Xiao R, Kim KH, Feng D, Lazar MA, Moore DD (2014) Nutrientsensing nuclear receptors coordinate autophagy. Nature 516: 112-115. doi:10.1038/nature13961

Man WC, Miyazaki M, Chu K, Ntambi J (2006) Colocalization of SCD1 and DGAT2: Implying preference for endogenous monounsaturated fatty acids in triglyceride synthesis. J Lipid Res 47: 1928-1939. doi:10.1194/ jlr.m600172-jlr200

Masuda M, Miyazaki-Anzai S, Keenan AL, Okamura K, Kendrick J, Chonchol M, Offermanns S, Ntambi JM, Kuro OM, Miyazaki M (2015) Saturated phosphatidic acids mediate saturated fatty acid-induced vascular calcification and lipotoxicity. J Clin Invest 125: 4544-4558. doi:10.1172/ jci82871

Masuda M, Miyazaki-Anzai S, Keenan AL, Shiozaki Y, Okamura K, Chick WS, Williams K, Zhao X, Rahman SM, Tintut Y, et al (2016) Activating transcription factor-4 promotes mineralization in vascular smooth muscle cells. JCI Insight 1: e88646. doi:10.1172/jci.insight.88646

Mochida K, Oikawa Y, Kimura Y, Kirisako H, Hirano H, Ohsumi Y, Nakatogawa H (2015) Receptor-mediated selective autophagy degrades the endoplasmic reticulum and the nucleus. Nature 522: 359-362. doi:10.1038/nature14506

Moulis M, Vindis C (2017) Methods for measuring autophagy in mice. Cells 6 : E14. doi:10.3390/cells6020014

Rahman SM, Baquero KC, Choudhury M, Janssen RC, de la Houssaye BA, Sun M, Miyazaki-Anzai S, Wang S, Moustaid-Moussa N, Miyazaki M, et al (2016) C/EBPbeta in bone marrow is essential for diet induced inflammation, cholesterol balance, and atherosclerosis. Atherosclerosis 250: 172-179. doi:10.1016/j.atherosclerosis.2016.03.040

Rahman SM, Schroeder-Gloeckler JM, Janssen RC, Jiang H, Qadri I, Maclean KN, Friedman JE (2007) CCAAT/enhancing binding protein beta deletion in mice attenuates inflammation, endoplasmic reticulum stress, and lipid accumulation in diet-induced nonalcoholic steatohepatitis. Hepatology 45: 1108-1117. doi:10.1002/hep.21614

Reue K, Leff T, Breslow JL (1988) Human apolipoprotein CIII gene expression is regulated by positive and negative cis-acting elements and tissuespecific protein factors. I Biol Chem 263: 6857-6864.

Schultz ML, Krus KL, Kaushik S, Dang D, Chopra R, Qi L, Shakkottai VG, Cuervo AM, Lieberman AP (2018) Coordinate regulation of mutant NPC1 degradation by selective ER autophagy and MARCH6-dependent ERAD. Nat Commun 9: 3671. doi:10.1038/s41467-018-06115-2

Seok S, Fu T, Choi SE, Li Y, Zhu R, Kumar S, Sun X, Yoon G, Kang Y, Zhong W, et al (2014) Transcriptional regulation of autophagy by an FXR-CREB axis. Nature 516: 108-111. doi:10.1038/nature13949

Shiozaki Y, Okamura K, Kohno S, Keenan AL, Williams K, Zhao X, Chick WS, Miyazaki-Anzai S, Miyazaki M (2018) The CDK9-cyclin T1 complex mediates saturated fatty acid-induced vascular calcification by inducing expression of the transcription factor CHOP. J Biol Chem 293 : 17008-17020. doi:10.1074/jbc.ra118.004706

Smith MD, Harley ME, Kemp AJ, Wills J, Lee M, Arends M, von Kriegsheim A, Behrends C, Wilkinson S (2018) CCPG1 is a non-canonical autophagy cargo receptor essential for ER-phagy and pancreatic ER proteostasis. Dev Cell 44: 217-232 e211. doi:10.1016/j.devcel.2017.11.024

Sterneck E, Zhu S, Ramirez A, Jorcano JL, Smart RC (2006) Conditional ablation of C/EBP beta demonstrates its keratinocyte-specific requirement for cell survival and mouse skin tumorigenesis. Oncogene 25: 1272-1276. doi:10.1038/sj.onc.1209144

Ueno T, Komatsu M (2017) Autophagy in the liver: Functions in health and disease. Nat Rev Gastroenterol Hepatol 14: 170-184. doi:10.1038/ nrgastro.2016.185

Xu X, Hu J, McGrath BC, Cavener DR (2013) GCN2 regulates the CCAAT enhancer binding protein beta and hepatic gluconeogenesis. Am J Physiol Endocrinol Metab 305: E1007-E1017. doi:10.1152/ajpendo.00063.2013

Zhang Y, Fang F, Goldstein JL, Brown MS, Zhao TJ (2015) Reduced autophagy in livers of fasted, fat-depleted, ghrelin-deficient mice: Reversal by growth hormone. Proc Natl Acad Sci U S A 112: 1226-1231. doi:10.1073/ pnas.1423643112

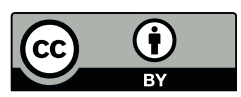

License: This article is available under a Creative Commons License (Attribution 4.0 International, as described at https://creativecommons.org/ licenses/by/4.0/). 